\title{
Selective Alkyne Hydrogenation over Nano-metal Systems: Closing the Gap between Model and Real Catalysts for Industrial Applications
}

\author{
Fernando Cárdenas-Lizana, Micaela Crespo-Quesada, and Lioubov Kiwi-Minsker
}

\begin{abstract}
The relationship between catalytic response and properties of the active phase is difficult to establish in classical heterogeneous catalysis due to the number of variables that can affect catalytic performance. Ultrahighvacuum surface methods applied to model catalyst surfaces are useful tools to assess fundamental issues related to catalytic processes but they are limited by the significant differences with catalysts in the working state. In an attempt to overcome this issue, (unsupported) nano-metal systems with controlled size and shape have been synthesized and tested in selective alkyne hydrogenation. The results revealed a dependency of nano-particles (NPs) morphology (size and shape) and allowed the identification of the active sites for this type of reaction. The nature of the stabilizer (steric and electrostatic stabilization) used in the NPs preparation has been shown to influence catalytic performance. The tailored active phase was subsequently immobilized on suitable nano- and micro-structured inorganic (e.g. 3D sintered metal fibers) supports with controlled surface properties in order to corroborate if the results obtained on the optimized nano-metal systems could be extrapolated to real catalysts. This article highlights the advantages and limitations of the analysis of selective alkyne hydrogenation over nano-metal systems that close the gap between model and real catalysts where the main challenges that lie ahead are summarized.
\end{abstract}

Keywords: Alkyne $\cdot$ Monodispersed colloids $\cdot$ Selective hydrogenation $\cdot$ Supported palladium catalyst

\section{Introduction/Scope}

Catalysis is crucial in green chemistry and vital for sustainable chemical processing. ${ }^{[1]}$ Hydrogenations are the most extensively applied catalytic reactions in the fine chemical industry. ${ }^{[2]}$ In order to optimize the performance of a specific catalyst formulation, both a combined fundamental understanding at molecular level and con-

${ }^{*}$ Correspondence: Prof. Dr. L. Kiwi-Minsker Ecole Polytechnique Fédérale de Lausanne Group of Catalytic Reaction Engineering Station 6

$\mathrm{CH}-1015$ Lausanne

Tel.: +4121693 3182

Fax: +41216933667

E-mail: lioubov.kiwi-minsker@epfl.ch sideration of catalyst design over multiple levels are required. The elucidation of the structural properties of metal catalyst surfaces is an extremely challenging task due to the involvement of micro and macroscopic areas with various compositions and phases. A supported metal catalyst differs significantly from a 'model' uniform reacting surface, displaying a distribution of interaction energetics associated with various exposed crystal faces, occurrence of dislocations and other disturbances. A combination of theoretical calculations ${ }^{[3]}$ and refined surface characterization techniques ${ }^{[4]}$ can hold the key in gaining a deeper understanding of catalyst structure and possible transition states/reaction pathways. However, theoretical evaluation is often speculative while experimental characterization techniques normally use high vacuum conditions and, therefore, catalyst structure under reaction conditions is often impossible to determine. Finding the most effective catalyst for a specific process calls for an initial optimization over model systems. This approach relies on carrying out catalytic reactions on ideal systems, such as metal single-crystal surfaces, under realistic conditions close to those typically found in industrial applications. This allows a systematic analysis of critical variables affecting the cata- lytic response. Once the model system has been optimized, the information obtained can be employed to design a more realistic supported metal catalyst. This transition is crucial since pressure and material gaps between supported metal catalysts (complex structure operating at high temperature and pressure) and model systems (idealized surface under vacuum) are significant. ${ }^{[5]}$ The last step in the full process design involves the integration of green chemistry and engineering in order to find the best catalyst/reactor system and optimum operation conditions.

Over the past few years, we have employed a methodology for the development of heterogeneous catalysts from nano-metal (colloidal) to real heterogeneous catalysts in order to make a desired industrial transformation technically feasible. In the following sections, the steps involved in this process are presented with a special emphasis given to the critical variables that determine the catalytic response. The selective $\mathrm{C} \equiv \mathrm{C}$ hydrogenation, with fundamental industrial importance, ${ }^{[6]}$ has been used as a test process for the application of both nano-metal colloids and real cata-

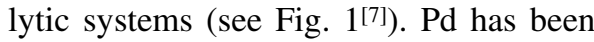
selected as the metal of choice due to its known enhanced selectivity towards olefin formation. ${ }^{6]}$ 


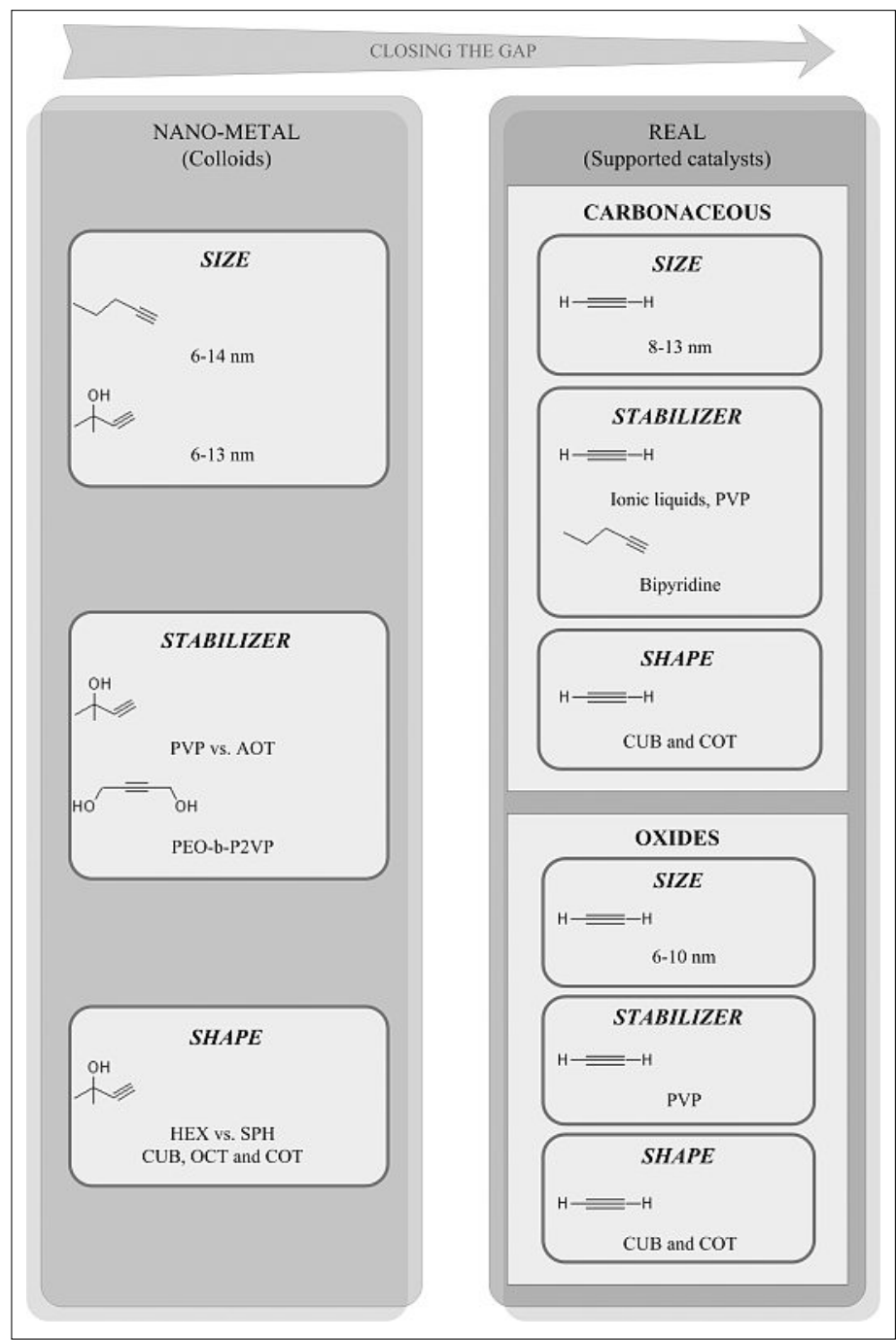

Fig. 1. Schematic flowchart showing the main stages involved in the catalyst optimization from nanometal to real systems.

\section{Nano-metals: A Bridge between Model and Real Catalysts}

The first definition of 'structure sensitivity' was provided back in 1969 by Boudart. ${ }^{[8]} \mathrm{He}$ proposed that, for some reactions, catalytic response was dependent on differences in the morphology of the active phase, i.e. nanoparticle shape and/ or size variations, normally in the range of 1-10 nm. Multiple carbon-carbon bond hydrogenations are known to be structure sensitive. ${ }^{[9]}$ However, the nature of this dependence is still a matter of debate. The roots of the problem can be found in the properties of the catalytic systems typically used for this type of reactions which are based on (oxide or carbonaceous) supported transition metal nanoparticles. Indeed, in this type of heterogeneous catalysts, metal crystal morphology is determined by a combination of factors, e.g. synthesis method, the nature of the metal precursor, activation conditions and/or the nature of the support, where the genesis of monodispersed metal catalysts with one specific crystallite shape is very difficult to achieve. This, in turn, means that it is almost impossible to establish an explicit correlation between catalytic performance and crystal morphology. Colloidal synthesis techniques based on electrostatic and/ or steric stabilization provide an attractive alternative for the preparation of nanometal particles with specific size and shape (Fig. 2aI-V). Since they are exempt of the typical perturbations found in supported catalysts, e.g. metal support interactions or mass-transfer limitations, they allow a direct analysis of the key properties of the active sites that control catalytic response. However, the analysis of the true catalytic behavior requires the removal of the stabilizer and/or capping agents used for the preparation.

Taking into account the advantages of the aforementioned methodology, we have considered the effect of Pd particle size in the liquid phase hydrogenation of hexyne. ${ }^{\text {[7a] }}$ Spherical Pd colloids with a range of particle sizes (6-14 nm) were synthesized by reverse microemulsion water/ AerosolOT/isooctane at different waterto-surfactant ratios. We observed that selectivity to the target 1-hexene was insensitive to particle size and equal to $96.5 \%$ (at $X=85 \%$ ). A greater (15-fold) hydrogenation rate per surface mol of $\mathrm{Pd}$, i.e. turnover frequency (TOF), with nanoparticle size (from 11-14 nm) was observed. Similar activity was obtained over colloids with $d_{p}>13 \mathrm{~nm}$, which was equivalent to that recorded for Pd black. The same correlation between catalytic response and metal dispersion was obtained in the selective liquid phase hydrogenation
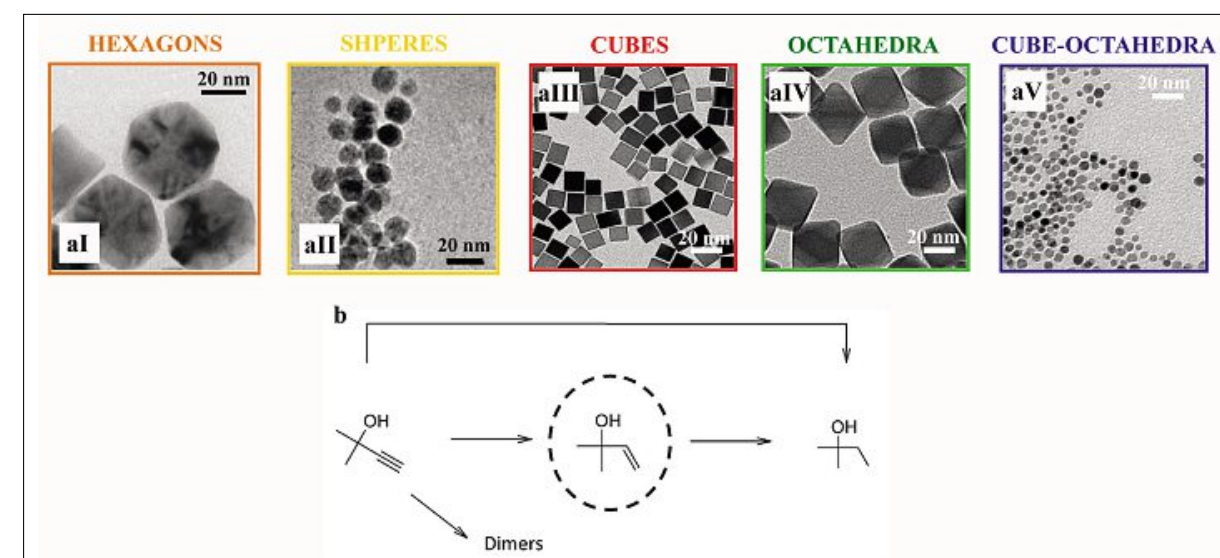

cI

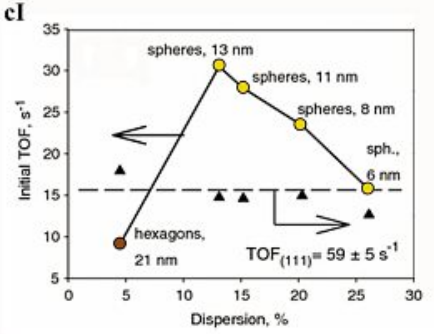

cII

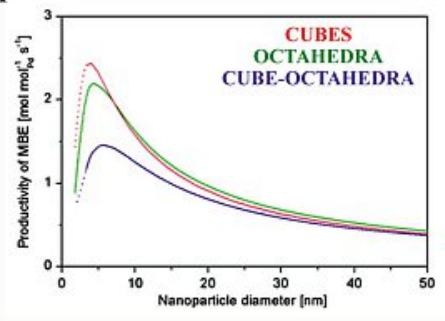

Fig. 2. (a) Representative HR-TEM images of stabilized Pd nanocrystals with (I) hexagonal (HEX), (II) spherical (SPH), (III) cubic (CUB), (IV) octrahedral (OCT) and (V) cubeoctahedral (COT) shape; (b) Reaction pathway and (c) associated catalytic response in terms of turnover frequency (TOF) as a function of morphology, shape and size, (II) HEX SPH and (II) CUB OCT COT) in the hydrogenation of 2-methyl-3-butyn-2-ol. Note: the target olefin 2-methyl-3-buten-2-ol product is framed. Adapted from refs [7c] and [7d]. 
of 2-methyl-3-butyn-2-ol (MBY) where a range of intermediates/products can be formed (Fig. 2b); selectivity to the olefin alcoholic product was found to be insensitive to Pd particle size whereas activity, expressed as initial TOF, increased (twofold) over larger particles $(6 \rightarrow 13 \mathrm{~nm})$. ${ }^{[7 \mathrm{~b}]}$ It was, however, striking to find that specific activity, i.e. expressed per $\mathrm{Pd}$ atoms on (111) planes, remained constant.

The shape of the colloidal nanoparticles determines the type of crystal plane exposed on the crystallite surface, e.g. cubes show exclusively $\operatorname{Pd}(100)$, octahedra present solely $\operatorname{Pd}(111)$ while a combination of both can be found in cubeoctahedra (sphere). We extended this work with the aim of analyzing in more depth the possibility of a structure-induced effect where modifications in crystallite shape can promote a distinct catalytic response, assuming that each surface atom exhibits a different reactivity. The liquid phase hydrogenation of MBY was investigated over monodispersed unsupported Pd nanohexagons (Fig. 2aI) and Pd nanospheres (Fig. 2aII) synthesized using a seed-mediated method. ${ }^{[7 c]}$ In order to identify the active sites responsible for the catalytic response, the number of atoms located on (111) facets as well as the total surface atoms were estimated using crystal surface atom statistics. ${ }^{[10]}$ The results generated confirmed $\operatorname{Pd}(111)$ atoms as the active sites for the catalytic reaction, i.e. independence of specific TOF, expressed per $\mathrm{Pd}(111)$ atoms, with particle size $(6-20 \mathrm{~nm})$ and shape (hexagon $v s$. sphere) (Fig. 2cI). The reaction was found to be size- and shape-independent, yet structure-sensitive. It is worth mentioning that the nanoparticles used in this work mainly bear $\operatorname{Pd}(111)$ bound clusters and so, the influence on catalytic response of other type of crystal planes, e.g. $\operatorname{Pd}(100)$, could have been overlooked. Because of this, the work was consequently complemented with the investigation of structure sensitivity in the MBY hydrogenation over welldefined poly (vinyl pyrrolidone) (PVP)stabilized cubic (Fig. 2aIII), octahedral (Fig. 2aIV) and cube-octahedral (Fig. 2aV) Pd nanoparticles synthesized via a solution-phase method. [7d] The activity/selectivity response suggested the involvement of two types of active site, namely, atoms located on either planes or edges. It was therefore proved that $\operatorname{Pd}(100)$ and $\operatorname{Pd}(111)$ are equally active in MBY hydrogenation. A two-site Langmuir-Hinshelwood model represents adequately the experimental (activity and selectivity) data allowing an accurate description of the reaction mechanism. Partial-hydrogenation to the target 2-methyl-3-buten-2-ol (MBE) product was found to take place on both types of sites. Activity response was found to be dependent on the coordination number where edge atoms were less active (factor of 4) relative to plane atoms for the selective semi-hydrogenation. In contrast, overhydrogenation (to 2-methyl-3-butan-2-ol (MBA)) was shown to occur exclusively on edge atoms, a result that we tentatively ascribed to an increased alkene adsorption strength on this type of sites. Kinetic simulations suggested PVP-stabilized cubic Pd nanoparticles with $d=3 \mathrm{~nm}$ as the optimum monometallic colloids for increased MBE production (Fig. 2cII).

In order to obtain thermodynamically stable morphology-controlled nanoparticles, the use of organic stabilizing and capping agents (or growth directing agents) is necessary. A strong effect on the electronic properties of the active phase can be expected for these substances which surround and are in intimate contact with the metal nanoparticles, although this interaction is still not well understood. We have analyzed the effect of the stabilizing agent with PVP and sodium di-2-ethylhexylsulfosuccinate (AOT), which act through steric and electrostatic stabilization, respectively. ${ }^{[7 e]}$ In the liquid phase hydrogenation of MBY, increased TOF but lower selectivity towards MBE was obtained over AOT-stabilized Pd colloids $\left(S_{A O T-P d X=50 \%}=\right.$ $90 \%$ vs. $S_{P V P-P d X=50 \%}=96 \%$ ). We tentatively associate this result with a stronger stabilizer-metal interaction in PVP-stabilized nanostructures. Indeed, depending on the properties of the stabilizing agent and the nature of the reaction, the former can have a negative effect on catalytic activity due to site blocking.

\section{Real Catalysts: Supported Pd Nano-particles}

As seen in the previous section, nanometal colloidal particles offer a unique op- portunity for the systematic analysis of the active sites and studying the mechanism of a catalytic reaction at molecular level. Industrial catalysts, on the other hand, are characterized by an active phase immobilized on a suitable carrier. Support materials provide a large catalytic surface area and prevent active phase sintering during operation. Furthermore, they facilitate both catalyst handling and separation from the reaction medium. In addition to this, the support can also act as a co-catalyst or affect catalytic performance through interactions with the active phase. Therefore, in the design of industrial catalysts for a given application, in addition to active phase morphology and nature of the stabilizer, support effects need to be taken into consideration as a critical factor affecting catalytic response.

Typical heterogeneous catalysts used in industry are based on pellets obtained by extrusion or high pressure compacting of powder systems characterized by a metal phase immobilized in a support. Structured supports, e.g. monoliths, membranes, foams and fibrous materials, offer a series of advantages with respect to powder catalysts as a result of the open macrostructures leading to low pressure drop during fluid-passage, narrow residence time distribution and enhanced mass and heat transfer. In particular, fibrous materials ${ }^{[11]}$ such as sintered metal fibers (SMF) of mechanically and chemically stable thin metal filaments offer additional benefits in terms of heat and mass transfer due to their high thermo-conductivity[12] and regular open structure, ${ }^{[13]}$ respectively (Fig. 3). The major drawback of fibrous supports is the low associated specific surface area (SSA). In order to overcome this limitation, the fibers can be homogeneously coated with a layer of a carrier with increased SSA.

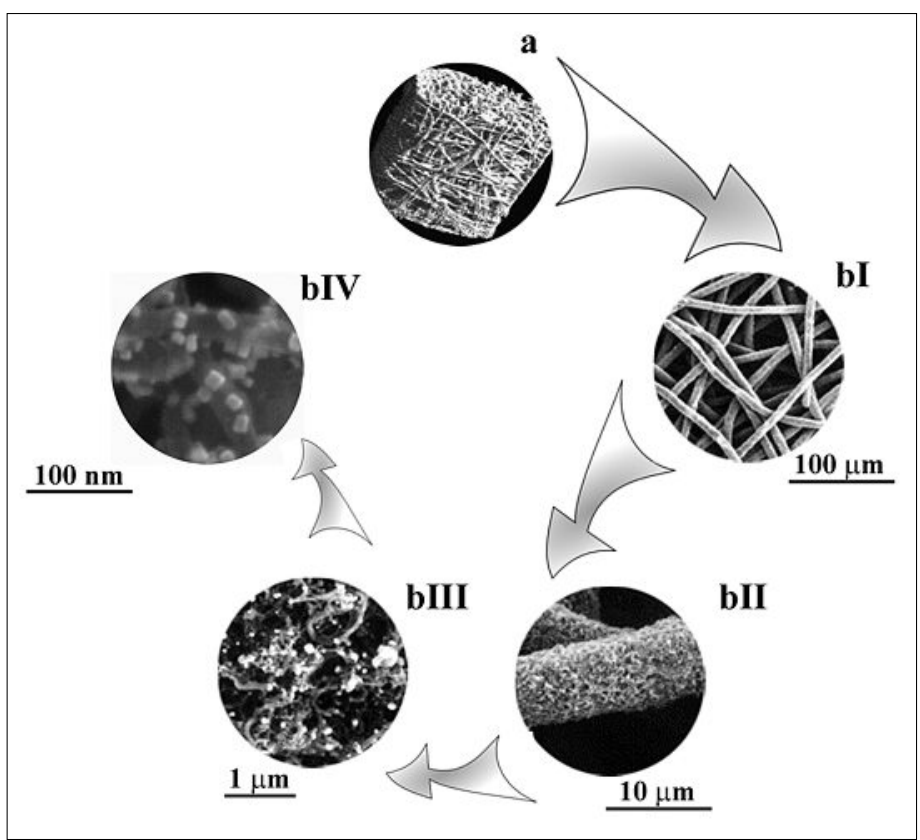

Fig. 3. (a) 3D tomography reconstruction and (b) SEM images of carbon nanofibers (CNF) supported cube Pd nanocrystals; (I) low, (II) medium and (III-IV) high magnification. 
Activated carbon is typically employed as a carbonaceous-based material due to the combination of large surface area $\left(\mathrm{SSA}>600 \mathrm{~m}^{2} \mathrm{~g}^{-1}\right)$, adsorption capacity, and cost effectiveness. ${ }^{[14]}$ Nonetheless, the significant micropore content of this material can result in physical transport constrains. Carbon nanofibers (CNF), on the other hand, can be directly grown on SMF to form a homogeneous and mesoporous layer of graphitic carbon. The research work of Tribolet et al..$^{[13,15]}$ on the growth of $\mathrm{CNF}$ from Inconel or stainless steel SMF filters resulted in the development of a composite material with a uniform CNF layer strongly anchored to the metal surface where the initial open structure of SMF filters was preserved. The catalytic potential of the novel $\mathrm{Pd} / \mathrm{CNF} / \mathrm{SMF}$ Inco was tested in the selective hydrogenation of acetylene, an extremely relevant reaction in the polyethylene industry. Higher TOF values were obtained over $\mathrm{Pd} / \mathrm{CNF} /$ $\mathrm{SMF}_{\text {Inconel }}$ relative to microporous activated carbon fibers supported $\mathrm{Pd}$ and ascribed to the more graphitic nature of CNF.[15]

Once the benefits of the newly developed structured support had been proven, and taking into account the knowledge obtained from the study of colloidal catalysts, we continued the catalyst optimization for industrial applications over supported systems. A series of CNF-based supported Pd catalysts were synthesized and employed for alkyne hydrogenation in order to elucidate the effects of particle size, shape and the nature of the stabilizers, as depicted in Fig. 1.

\subsection{Size and Shape Effects}

The results presented in section 2 for liquid-phase alkyne hydrogenation over unsupported Pd nanocolloids are consistent with an antipathetic structure-sensitivity, i.e. TOF increases with nanoparticle size. In order to analyze if the same trends apply to gas-phase operation we selected the hydrogenation of acetylene as model reaction. Ruta et al. ${ }^{[7 \mathrm{f}]}$ analyzed nanoparticle size-effect over CNF/SMF supported monodispersed Pd nanoparticles of 8, 11, and $13 \mathrm{~nm}$. TOF was found to increase (from $15 \mathrm{~s}^{-1}$ up to $24 \mathrm{~s}^{-1}$ ) for the larger $\mathrm{Pd}$ nanoparticles where a diameter of $11 \mathrm{~nm}$ was found to be critical above which the nanoparticles specific activity is not sizedependent. This result is similar to that obtained in the hydrogenation of 1-hexyne over unsupported colloids ${ }^{[7 \mathrm{a}]}$ and suggests a similar behavior of both nanometal and real catalysts, in gas- and liquid-phase operation.

Shape and size effects for real catalysts were systematically investigated over CNF supported cubes $(6$ and $10 \mathrm{~nm})$ and cubeoctahedra $(5.5 \mathrm{~nm}) \mathrm{Pd}$ nanoparticles in the selective hydrogenation of acetylene.
In terms of Pd nanoparticle size, the results obtained were again consistent with an antipathetic structure sensitivity. ${ }^{[7 f]}$ Regarding shape, we found increased activity over cube-octahedral nanoparticles $\left(\mathrm{TOF}_{111}=1622.7 \pm 81.1 \mathrm{~s}^{-1} ; \mathrm{TOF}_{100}=\right.$ $361.5 \pm 9.4 \mathrm{~s}^{-1}$ and $\left.\mathrm{TOF}_{\text {edge }}=92.8 \pm 4.4 \mathrm{~s}^{-1}\right)$ compared to cubic crystallites with similar dispersion. This result deviates from that obtained in the liquid-phase hydrogenation of MBY over model systems (see section 2) where we concluded that both plane atoms $(\operatorname{Pd}(100)$ and $\operatorname{Pd}(111))$ were equally active and where edge atoms, also involved in the catalysis, are considerably less active. ${ }^{[7 d]}$ Nonetheless, this observation could be an indirect evidence of a different structuresensitive behavior in gas phase operation as a result of $\mathrm{C}$-containing species deposition. ${ }^{[16]}$ Indeed, different Pd crystals facets have been shown to have distinct activities in the essential carbon laydown process during alkyne hydrogenation. ${ }^{[17]}$ This layer is formed in the early stages of the reaction and prevents the dissolution of hydrogen in the bulk of the nanoparticles. This hydrogen is known to be highly energetic and non-selective to olefin formation. ${ }^{[17,18]}$ The hydrogen diffusion-blocking effect ensures that only surface hydrogen, much less active but more selective, is available for the reaction. It has been demonstrated both experimentally ${ }^{[17]}$ and theoretically, ${ }^{[19]}$ that carbon diffusion into the bulk of $\mathrm{Pd}(111)$ is energetically unfavorable. Therefore, we envisioned a less pronounced carbon laydown process on cube-octahedral (with $\operatorname{Pd}(111)$ and $\operatorname{Pd}(100)$ ) relative to cubic (only Pd(100)) nanoparticles which could account for the differences in the recorded catalytic response.

\subsection{Effect of the Stabilizer}

At this point, we have established that differences in the stabilizing agents can impact on the activity $\left.{ }^{[7]}\right]$ and selectivity ${ }^{[20]}$ of unsupported nanoparticles for alkyne hydrogenation. In this section, we present the analysis extended to real (supported Pd-based) catalysts where the hydrogenation of acetylene has been selected as model process. A series of Pd nanoparticles were synthesized in situ in ionic liquids ([bmim] $\left[\mathrm{PF}_{6}\right]$, or $\left.[\mathrm{bmimOH}]\left[\mathrm{TF}_{2} \mathrm{~N}\right]\right)$ and further immobilized on CNF/SMF. ${ }^{[7 \mathrm{~g}]}$ The catalysts showed good long-term stability and did not promote any formation of green oil, the main undesirable reaction by-product. Such a response suggests that the IL cation-anion network surrounding the nanoparticles suppressed the formation of active-site ensembles which are known to catalyze the oligomerization of acetylene to green oil. Catalytic response can also be effectively modified by the removal of the stabilizing agent. However, in spite that a number of techniques have been suggested in the literature, e.g. plasma cleaning or thermal treatment, they either require sophisticated equipment or, in most of the cases, compromise the integrity of the nanoparticle morphology. We have shown that treatment by UV-ozone (UVO) is a suitable and mild technique for the removal of trace amounts of stabilizing agent in CNF/SMF supported PVPstabilized Pd nanoparticles where the size and shape of the crystals is maintained.[21] The amount of PVP on the surface during UVO cleaning was monitored by analyzing the $\mathrm{N}$ (from PVP) surface content by ex situ XPS analysis. After $4 \mathrm{~h}$ under UVO, cleaning of nitrogen was achieved on the sample. The removal of PVP resulted in a concomitant increase in activity (fourfold) and undesired green oil formation. We also demonstrated the key role of the stabilizer in liquid phase operation; Crespo-Quesada and co-workers ${ }^{[7 \mathrm{~h}]}$ used 2,2'-bipyridine (bipy) and an imidazolium tagged bipy ligand with $\mathrm{N}$-containing ligands for the synthesis of $\mathrm{Pd}$ nanoparticles. These ligands bear a double function; firstly, stabilizing agents to prevent the agglomeration of the nanoparticles during synthesis, and secondly, permanent catalyst modifier. The synthesized Pd nanoparticles were subsequently deposited on CNF/SMF supports and tested in the liquid-phase hydrogenation of 1-hexyne. The catalysts were found to be significantly more selective to the target olefin, even at full conversion, than an analogous reference catalyst with unmodified, i.e. $\mathrm{N}$-containing ligands in the stabilizer, Pd nanoparticles.

\subsection{Support Effects}

Besides carbon, oxides are the carriers normally employed for catalytic applications. The chemical and structural properties of these materials, e.g. acid-base and/or redox properties, crystallographic phase, can control the catalytic response in alkyne hydrogenation through metal-support interactions. The redox characteristics of the oxide support are crucial where partial reduction of carrier can result in i) partial/total blockage of the active site via migration of suboxide species and encapsulation of the metal particles and/or ii) the formation of new bimetallic phase(s) with modified electronic and geometric properties affecting catalytic performance in the hydrogenation of alkynes. We have shown the formation of a $\mathrm{PdZn}$ phase after reductive treatment of $\mathrm{Pd}$ nanoparticle on $\mathrm{ZnO}$ supports. The formation of this new phase was deemed responsible for the increased selectivity to the target alkene in the hydrogenation of MBY.[22] Fig. 4 shows SEM images of CNF (a) and $\mathrm{ZnO}$ (b) coated SMF supports. We compared the catalytic response of $\mathrm{Pd}$ nanoparticles supported on both, $\mathrm{CNF}$ and $\mathrm{ZnO}$, in the 


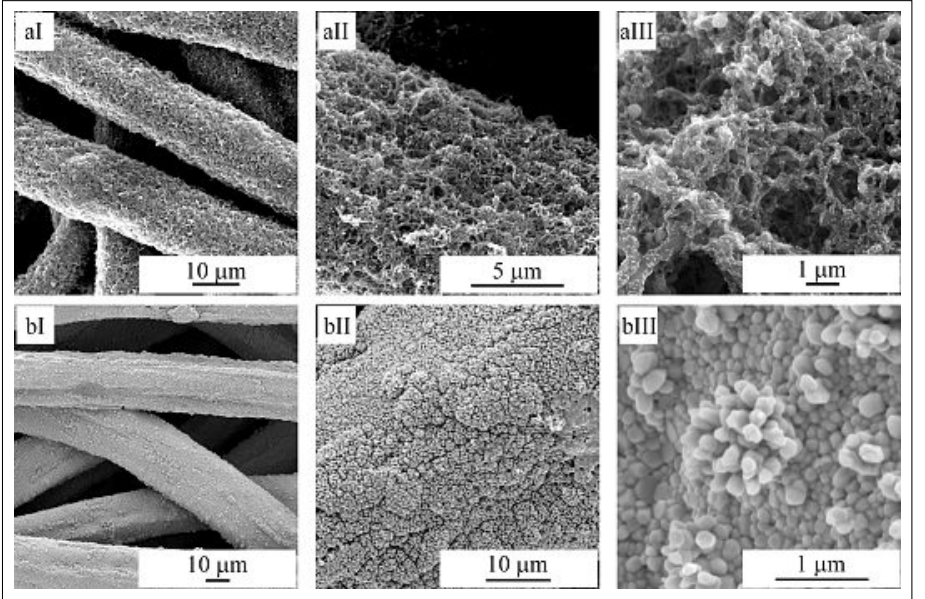

Fig. 4. SEM micrographs of (a) carbon nanofibers (CNF) and (b) ZnO-coated sintered metal fiber (SMF) supports; (I) low, (II) medium and (III) high magnification.

hydrogenation of acetylene. Lower activity but a measurable increase in olefin selectivity was recorded over $\mathrm{Pd} / \mathrm{ZnO} / \mathrm{SMF}$ that is consistent with the results obtained in the hydrogenation of MBY. The distinct catalytic behavior has been ascribed to the reducibility of $\mathrm{ZnO}$ which allows the formation of an intermetallic PdZn compound on the surface of the catalyst, as suggested by XPS analysis.

\section{Reactor Design}

In any catalytic route, after having developed the optimum catalyst for a specific industrial application, one last step must be performed to fully optimize the process. At this point, and in order to intensify the catalytic process, the most efficient catalytic material must be coupled with optimal reactor design and operating conditions. ${ }^{23]}$ The characteristics of the reactor in which the catalyst is bound to operate are crucial since they can control the outcome performance. Industrial catalytic hydrogenations are commonly carried out either in fixed-bed or suspension reactors, such as fluidized beds and mechanical stirred tank reactors. ${ }^{[23 a]}$ Among the main artefacts associated with conventional slurry stirred tank reactions, it is worth highlighting the influence of mass and heat transfer on chemical kinetics. External and internal gas-liquid and liquid-solid mass transfer often control the reaction kinetics leading to lower selectivity and productivity. In hydrogenation reactions, in particular, reliable estimation of mass transfer coefficients is difficult to achieve due to the challenging task of monitoring hydrogen concentration in the liquid phase. In order to tackle this issue, we used a Fugatron analyzer to estimate both internal and external mass transfer influences and obtain the intrinsic kinetics of MBY hydrogenation over Lindlar's catalyst. [24]

A second issue in slurry reactors, as compared to conventional fixed bed reac- tors, is catalyst abrasion and recovery. To overcome the drawbacks associated with classic heterogeneous catalysts based on powder supported metal systems, structured catalysts have been employed.[11] These novel catalysts are characterized by high porosities that result in low pressure drops. Furthermore, the open cross-flow structure of arranged catalysts facilitates intensive radial mixing and leads to narlar reactors. ${ }^{[23 a]}$ Considering these advantages, we developed a structured SMF based catalyst, i.e. $\mathrm{Pd} / \mathrm{ZnO} / \mathrm{SMF}$, for the same reaction. ${ }^{[15,22]}$ The catalyst was subsequently placed in a cage stirrer and used in a semi-batch reactor to study the kinetics of MBY hydrogenation. Our results demonstrate that the newly designed catalyst outperformed the standard Lindlar's catalyst where the formation of $\mathrm{PdZn}$ following a high temperature reduction not only increased activity but also selectivity to the target olefin.

One limitation of these structured systems is the amount of catalyst that can be integrated in a stirrer. Moreover, the mass of catalyst per volume diminishes with scale-up leading to reduced specific reactor performances. ${ }^{[25]}$ A loop reactor with an integrated catalytic bubble column staged with catalyst layers enables higher catalyst packing as compared to a standard stirred tank reactor. We tested a structured activatrow residence time distributions in tubu-

ed carbon fibers (ACF) supported Pd catalyst in the hydrogenation of 2-butyne-1,4diol. Selectivity to the semi-hydrogenation product of $97 \%$ was obtained at high conversions $(80 \%)$ where the catalyst showed remarkable stability (up to 6 months) with more than $375 \mathrm{~h}$ on stream.

The use of a loop reactor in complex reaction processes, such as the hydrogenation of alkynes, allows partial control of the high exothermicity for this type of reaction. However, complete backmixing leads to considerable losses in product yield at high conversions. Therefore, we developed a new reactor based on a bubble column staged with structured catalytic layers (SBCR) with integrated cross flow micro-heat-exchangers (MEX) and tested it in the solvent free hydrogenation of MBY.[26] The attained specific reactor performance was found to be several orders of magnitude above the values reported for conventional multiphase reactors where adjustment of the hydrogen partial pressure can increase the productivity up to the limits governed by the heat transfer performance of the MEX elements.

Conventional contactors present several problems for fast and highly exothermic hydrogenation reactions. In contrast, microreactors are a suitable alternative since mass and heat transfer processes can be accelerated by more than one order of magnitude. ${ }^{[23 a]}$ Nonetheless, in this type of configuration the introduction of the catalyst can be problematic, causing high pressure drops or catalyst maldistributions. Therefore, we adopted the concept of 'slit microreactor'. [27] The novel design as a structured catalytic wall microreactor (SWMR) allows the reaction mixture to flow through an empty micro-slit above a structured catalyst along the wall of the reactor (Fig. 5). A structured catalyst based on supported Pd nanoparticles embedded in ionic liquids, as the optimum formulation from the results obtained over model and real catalysts was used in the hydrogenation of acetylene. A selectivity of $70.5 \%$ was obtained at $96.5 \%$ conversion where no deactivation was observed during several hours on stream.

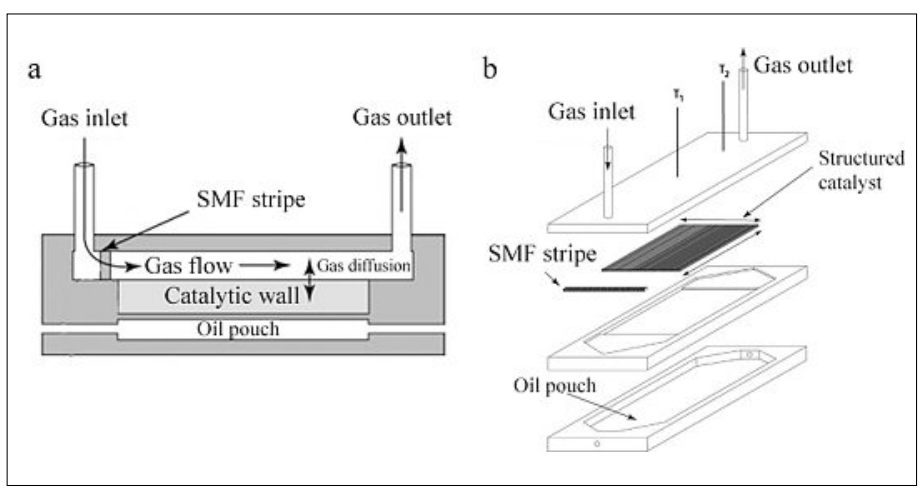

Fig. 5. (a) 2D and (b) 3D scheme of structured catalytic wall microreactor (SWMR). Adapted from ref. [27] with permission from Elsevier. 


\section{Conclusions and Future Remarks}

Surface analysis methodologies allow the exploration of uniform reacting surfaces at the atomic scale and can contribute to gaining a better understanding of catalyst structure and reaction pathways. Although these techniques have matured enormously over the last few decades, there is still a large gap that separates idealized surfaces under vacuum and complex catalysts operating at increased temperatures and pressures. Therefore, it is still not clear to what extent the information obtained from these 'model' systems can be extrapolated to real industrial catalysts. Nano-metal colloidal particles that combine optimum size and shape design have a crucial role to play in closing the gap between model and real catalysts, as it has been demonstrated in this Review. They provide a valuable means to identify the nature of the active sites for a specific industrial reaction, where the knowledge gained can be effectively applied to the design of real industrial catalysts.

This review has highlighted the significant differences for alkyne hydrogenation in activity and product distribution depending on the morphology (shape and size) and nature of the stabilizing agent. Future work should be directed at extending the type of stabilizing and capping agents and to consider a wider range of nano-metal sizes $(<4 \mathrm{~nm})$ and shapes in order to clearly identify the main parameters that control the catalytic response in a specific industrial process. Moreover, further research in alternative techniques for the removal of trace amounts of these agents, affecting catalytic response, without compromising the size and shape of the nano-metals is required.

Control over catalytic performance and catalyst reusability is crucial in order to implement a sustainable catalytic unit operation where the active site (molecular level) optimization has to be coupled with analysis in the macro- and meso-levels. At present, the work conducted on catalytic alkyne hydrogenation has been mainly focused exclusively on one of the three levels. An analysis of catalysts to promote alkyne hydrogenation taking into account the full process (from active site to reactor design and optimum operation conditions) should be conducted simultaneously to arrive at the best practical option.

Structured fibrous materials offer a series of advantages when compared to standard powder heterogeneous catalysts. However, they are limited by the amount of metal that can be loaded as a result of low SSA associated with the deposited support coating layer. Further investigation on alternative materials and techniques for deposition of this homogeneous layer is needed. Liquid-phase hydrogenation is largely limited to batch operation and reactor development for continuous $\mathrm{C} \equiv \mathrm{C}$ hydrogenation with heat removal as a means of process control is an important step forward in practical application. A further progressive approach is to harness the catalyst within the reactor structure by means of, for example, wall coating. In this case, investigation of alternative materials that can be easily incorporated and removed from the reactor is warranting.

\section{Acknowledgements}

The authors are grateful to Prof. Albert Renken, Dr. Natalia Semagina, Dr. Eric Joanet, Dr. Pascal Tribolet, Dr. Marina Ruta, Dr. Martin Grasemann, Dr. Brian Bromley, Dr. AnneLaure Dessimoz, Dr. Jean-Michel Andanson, Dr. Madvhanand Kashid, Andrea Bruehwiler and Artur Yarulin for their invaluable contributions to this work. Financial support over multiple years from the Swiss National Science Foundation is highly appreciated.

Received: June 22, 2012

[1] P. Anastas, N. Eghbali, Chem. Soc. Rev. 2010, 39, 301

[2] H.-U. Blaser, Catal. Today 2000, 60, 161.

[3] J. K. Nørskov, T. Bligaard, J. Rossmeisl, C. H. Christensen, Nat. Chem. 2009, 1, 37.

[4] M. Che, J. C. Védrine, 'Characterization of Solid Materials and Heterogeneous Catalysts - From Structure to Surface Reactivity', WileyVCH: Weinheim, 2012.

[5] M. A. Keane, J. Mater. Sci. 2003, 38, 4661.

[6] Á. Molnár, A. Sárkany, M. Varga, J. Mol. Catal. A: Chem. 2001, 173, 185.

[7] a) N. Semagina, A. Renken, L. Kiwi-Minsker, J. Phys. Chem. C 2007, 111, 13933; b) N. Semagina, A. Renken, D. Laub, L. KiwiMinsker, J. Catal. 2007, 246, 308; c) N. Semagina, L. Kiwi-Minsker, Catal. Lett. 2009,
127, 334; d) M. Crespo-Quesada, A. Yarulin, M. Jin, Y. Xia, L. Kiwi-Minsker, J. Am. Chem. Soc. 2011, 133, 12787; e) L. Kiwi-Minsker, M. Crespo-Quesada, Top. Catal. 2012, 55, 486; f) M. Ruta, N. Semagina, L. Kiwi-Minsker, $J$. Phys. Chem. C 2008, 112, 13635; g) M. Ruta, G. Laurenczy, P. J. Dyson, L. Kiwi-Minsker, $J$. Phys. Chem. C 2008, 112, 17814; h) M. CrespoQuesada, R. R. Dykeman, G. Laurenczy, P. J. Dyson, L. Kiwi-Minsker, J. Catal. 2011, 279, 66.

[8] M. Boudart, Adv. Catal. 1969, 20, 153.

[9] M. Crespo-Quesada, F. Cárdenas-Lizana, A.-L. Dessimoz, L. Kiwi-Minsker, 2012, ACS Catal. 2012, 2, 1773.

[10] R. Van Hardeveld, F. Hartog, Surf. Sci. 1969 15, 189.

[11] L. Kiwi-Minsker, Chimia 2002, 56, 143

[12] D. R. Cahela, B. J. Tatarchuk, Catal. Today 2001, 69, 33.

[13] P. Tribolet, L. Kiwi-Minsker, Catal. Today 2005, 102, 15.

[14] F. Rodríguez-Reinoso, Carbon 1998, 36, 159.

[15] P. Tribolet, L. Kiwi-Minsker, Catal. Today 2005, 105, 337

[16] a) S. Abellò, D. Verboekend, B. Bridier, J Pérez-Ramírez, J. Catal. 2008, 259, 85; b) B. Bridier, J. Pérez-Ramírez, J. Am. Chem. Soc. 2010, 132, 4321.

[17] D. Teschner, E. Vass, M. Hävecker, S. Zafeiratos, P. Schnörch, H. Sauer, A. KnopGericke, R. Schlögl, M. Chamam, A. Wootsch, A. S. Canning, J. J. Gamman, S. D. Jackson, J. McGregor, L. F. Gladden, J. Catal. 2006, 242, 26.

[18] M. W. Tew, M. Janousch, T. Huthwelker, J. A. van Bokhoven, J. Catal. 2011, 283, 45.

[19] L. Gracia, M. Calatayud, J. Andres, C. Minot, M. Salmeron, Phys. Rev. B 2005, 71.

[20] N. Semagina, E. Joannet, S. Parra, E. Sulman, A. Renken, L. Kiwi-Minsker, Appl. Catal. A: Gen. 2005, 280, 141

[21] M. Crespo-Quesada, J.-M. Andanson, A Yarulin, B. Lim, Y. Xia, L. Kiwi-Minsker, Langmuir 2011, 27, 7909.

[22] a) M. Crespo-Quesada, M. Grasemann, N. Semagina, A. Renken, L. Kiwi-Minsker, Catal. Today 2009, 147, 247; b) N. Semagina, M. Grasemann, N. Xanthopoulos, A. Renken, L. Kiwi-Minsker, J. Catal. 2007, 251, 213.

[23] a) A. Renken, L. Kiwi-Minsker, in 'Advances in Catalysis', Academic Press.: New York, 2010; Vol. 53; b) L. Kiwi-Minsker, M. CrespoQuesada, Chimia 2011, 65, 699.

[24] A. Bruehwiler, N. Semagina, M. Grasemann, A. Renken, L. Kiwi-Minsker, A. Saaler, H Lehmann, W. Bonrath, F. Roessler, Ind. Eng. Chem. Res. 2008, 47, 6862.

[25] L. Kiwi-Minsker, E. Joannet, A. Renken, Chem. Eng. Sci. 2004, 59, 4919.

[26] M. Grasemann, A. Renken, M. Kashid, L. KiwiMinsker, Chem. Eng. Sci. 2010, 65, 364.

[27] L. Kiwi-Minsker, M. Ruta, T. Eslanloo-Pereira, B. Bromley, Chem. Eng. Process. 2010, 49, 973. 